\section{A) Check for updates}

Cite this: Food Funct., 2019, 10, 6322

\title{
Dose-response relationship between cocoa flavanols and human endothelial function: a systematic review and meta-analysis of randomized trials $\uparrow$
}

\author{
Ye Sun, ${ }^{a}$ Diane Zimmermann, (D ${ }^{\mathrm{b}}$ Carlos Antonio De Castro ${ }^{\mathrm{a}}$ and \\ Lucas Actis-Goretta (D) *a
}

\begin{abstract}
Background: Several intervention studies have investigated the relationship between cocoa flavanols and endothelial function. However, the shape of the association and the type of compounds responsible for the effects are largely unknown. Objective: To examine the dose-response association between the consumption of cocoa flavanols and endothelial function, measured by flow-mediated dilation (FMD). Design: Two investigators searched Scopus ${ }^{\circledR}$ for the relevant human intervention studies, which were pooled and meta-analysed. Heterogeneity in the findings was explored with various subgroup analyses. Results: Fifteen published articles with 18 intervention arms met the inclusion criteria. Participants in these intervention groups received 80 to $1248 \mathrm{mg}$ (mean: $704 \mathrm{mg}$ ) more flavanols than control groups. A significant improvement of FMD by $1.17 \%(95 \% \mathrm{Cl}$ : $0.76 \%$ to $1.57 \%)$ was calculated, with strong evidence of a non-linear association (inverted $U$-shape) between cocoa flavanols and FMD. Conclusions: This meta-analysis provides evidence that cocoa flavanols could significantly improve endothelial function, with an optimal effect observed with $710 \mathrm{mg}$ total flavanols, $95 \mathrm{mg}(-)$-epicatechin or $25 \mathrm{mg}(+)$-catechin. However, there was substantial variation in the results that could not be explained by the characteristics that we explored, and there were significant risk-of-bias concerns with a large majority of the studies.
\end{abstract}

\author{
Received 1st August 2019 \\ Accepted 28th August 2019 \\ DOI: 10.1039/c9fo01747j \\ rsc.li/food-function
}

\section{Introduction}

Observational studies suggest that consumption of flavanol-rich products, such as dark chocolate, is associated with a reduced risk of developing cardiovascular disease. ${ }^{1,2}$ Additionally, a number of human randomized clinical trials (RCTs) have convincingly shown a vascular benefit from consumption of flavanol-rich products in different population groups. Meta-analyses of such clinical trials have demonstrated that consumption of flavanol-rich products improved endothelial dysfunction as assessed by flow-mediated dilation (FMD). ${ }^{3,4}$

Whilst, from a nutritional perspective, it is often more meaningful to investigate the impact of foods rather than nutrients, ${ }^{5}$ sometimes nutrient analysis is necessary to gain insight into the main bioactive constituents, in order to be able to recommend foods comparably rich in the most prominent bioactive(s). ${ }^{6}$ Nutrient analysis of cocoa flavanols shows

\footnotetext{
${ }^{a}$ Nestlé Research Centre Singapore, Singapore.

E-mail: lucas.actisgoretta@rdsg.nestle.com; Tel: +65 66352242

${ }^{b}$ Nestlé Research Centre Lausanne, Switzerland

$\dagger$ Electronic supplementary information (ESI) available. See DOI: 10.1039/ c9fo01747j
}

that they comprise several types of compounds such as (-)-epicatechin, $(+)$-catechin and their oligomers, called procyanidins (degree of polymerization from $n=2$ to $n=10$ ). ${ }^{7}$ Although few studies have documented the generation of absorbable compounds by microbiological biotransformation of procyanidins in the large intestine ${ }^{8}$ most evidence suggests that essentially monomers and, to a very low extent dimers, are absorbed in the small intestine; ${ }^{7}$ among them, (-)-epicatechin would be primarily responsible for their biological activity.

Acute intervention trials have demonstrated improvement in FMD after consumption of 1-2 $\mathrm{mg}$ per $\mathrm{kg}$ body weight (up to $200 \mathrm{mg}$ ) of purified (-)-epicatechin ${ }^{9,10}$ while a 30-day intervention trial with $100 \mathrm{mg}$ of purified (-)-epicatechin failed to show any improvement in the same parameter. ${ }^{11}$ In a previous meta-analysis, Hooper et al. $2012^{3}$ observed improvement in FMD by chocolate/cocoa consumption both acutely and chronically, with no apparent dose-effect relationship.

A number of articles investigating this area have been published since then and, with this background, we aimed to perform an updated systematic review and meta-analysis to summarize the current scientific evidence, and evaluate the main bioactive(s) and their optimal doses from cocoa/chocolate to positively influence endothelial function assessed by FMD. 


\section{Subjects and methods}

\section{Data sources and search strategy}

A literature search was performed independently by two investigators by means of the Scopus ${ }^{\circledR}$ database (Elsevier BV, Amsterdam, The Netherlands), to identify RCTs published up to January 2019 that investigated the effects of daily consumption of flavanol-rich cocoa products on endothelial function. Key words used in the search criteria were:

("Arterial function" OR "vascular function" OR "vascular dysfunction" OR "flow mediated dilat*" OR "flow mediated vasodilat*" OR "endothel* dependent dilat*" OR "endothel* dependent vasodilat*" OR flow-mediated OR "flow mediated" OR brachial reactivity OR FMD) AND (cocoa* OR chocolate OR cacao) AND (random* control* trial OR control* clinical trial OR trial OR "double blind*" OR "single blind*")

In addition, reference lists of published trials and reviews were checked.

\section{Literature selection}

RCTs were included in the meta-analysis if they met the following inclusion criteria: the primary or secondary outcome of the study was FMD; the intervention lasted for at least 1 week; the intervention was a flavanol-containing cocoa/chocolate food; the last measurement of FMD was performed in a fasted condition after the intervention period. A flow diagram for study selection is presented in Fig. 1.

\section{Data extraction}

Following the literature search, the investigators reviewed the study titles and abstracts followed by the full-text articles for eligibility. Discrepancies were resolved by discussion and agreement. Data were extracted following the inclusion criteria; studies were also excluded if there was no FMD measurement, no control or comparator group, did not include cocoa or chocolate extract, or when acute intervention was performed. Data on means and standard deviations (SD) at the end of the intervention for both treatment and control groups were extracted. If FMD data were not available in the text, they were estimated from the plots. If SDs were not reported, they were calculated or estimated from standard errors (SEs), confidence intervals (CIs), $p$-values for difference in means, or pooled correlation coefficients between baseline and final measurements from trials reporting sufficient information. Multiple comparison arms sharing the same control group were combined to create a single pair-wise comparison to avoid double counting and correlated comparisons. If more than one flavanol dose levels were tested, only the results from the highest dose arm were included in the main meta-analysis, but all arms with different doses were included in the subgroup meta-analysis by dose of flavanols and in the dose-response analysis. If more than one treatment time was described in the intervention (i.e. 7 and 14 days), only the longest intervention time was considered for the main meta-analysis, but all time-points were used in the subgroup meta-analysis grouped by duration of treatment.

\section{Risk of bias assessment}

To assess the risk of bias, two investigators independently performed a risk-of-bias assessment using the Cochrane Collaboration's tool. ${ }^{12}$ The criteria to assess risk of bias were random sequence generation, allocation concealment, blinding of participants and personnel, blinding of outcome assessors, incomplete outcome data or selective reporting. Overall risk of bias was defined as low when 4-6 of these criteria scored low risk and 0-2 criteria scored unclear risk. Moderate risk of bias was defined as unclear risk in 3-4 of the criteria, low risk in $2-3$ criteria, and moderate risk in $0-1$ criteria. Trials with high risk assessment in any of the criteria were considered as high risk.

\section{Statistical analysis}

All statistical analyses for the meta-analysis were performed using the open-source statistical software $\mathrm{R}$ ver. 3.2.3 and Stata version 15 (StataCorp LLC, Texas, USA). The meta-analysis was performed using the function metacont () within the package meta which produces both fixed- and random-effects estimates with continuous outcome data. Inverse variance weighting was used to pool the different studies/publications. ${ }^{13}$ Results from the random-effects model were presented, considering the

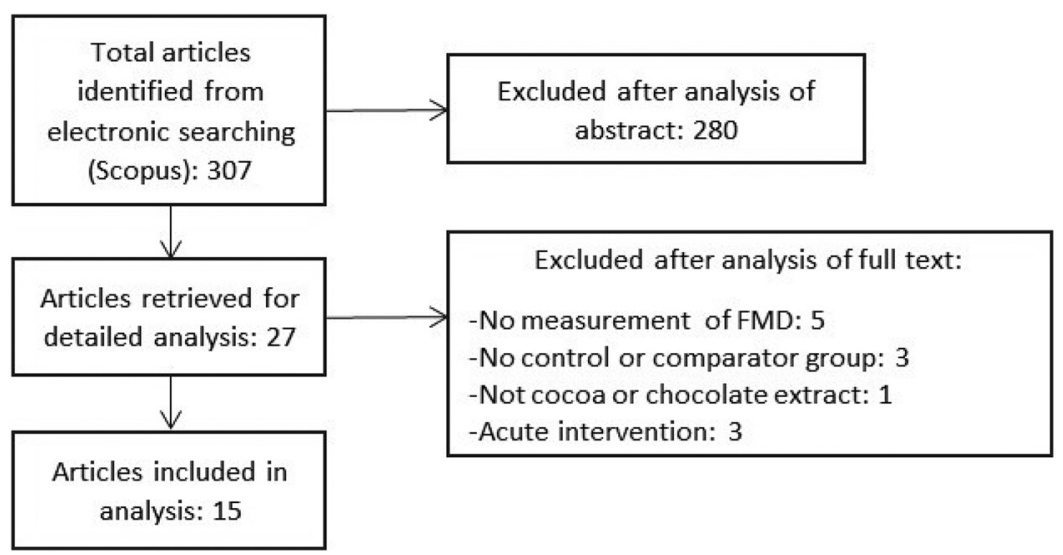

Fig. 1 PRISMA flow chart of study selection. 
large between-study heterogeneity in most cases. Sensitivity analyses were conducted with a fixed-effects model to compare different models. The graphical representation and smoothing curve were done with package ggplot $2^{14,15}$ with the function geom_smooth().

Locally weighted scatterplot smoothing (loess) function was used since the number of observations was below 1000 . Weights were applied using the ones from the random-effects model of the meta-analysis (due to relatively high heterogeneity). Heterogeneity in the study results was quantified by the $I^{2}$ statistic. ${ }^{14}$ Potential sources of heterogeneity were investigated by stratified meta-analyses according to various study characteristics defined a priori: study design, subject characteristics, type of intervention, duration of intervention, risk of bias, and funding body.

Meta-regression was used to assess the statistical significance of the differences across strata. Residual heterogeneity was also assessed using $I^{2}$ after each of the above-mentioned characteristics were accounted for in meta-regression. Sensitivity analyses were performed to assess the robustness of the meta-analysis by removing the studies one at a time. Publication bias was assessed by visual inspection of the funnel plot, ${ }^{16}$ by Begg's test ${ }^{17}$ and Egger's test. ${ }^{18}$

\section{Results}

\section{Study characteristics}

The literature search identified 307 articles of which 15 human clinical trials met the inclusion criteria (Fig. 1). The trials included 730 participants in 18 different intervention arms. ${ }^{19-33}$ The characteristics of these clinical trials are displayed in Table 1 . Nine trials had cocoa drink intervention, five investigated chocolate as intervention and the remaining one had a combination of cocoa drink plus chocolate intervention. Trial intervention duration ranged from 7 to 84 days (median: 28 days). The level of flavanol intake in these studies ranged from 80 to $1248 \mathrm{mg}$ (mean: $704 \mathrm{mg}$ ).

\section{Endothelial dysfunction assessed by FMD after ingestion of cocoa drink, chocolate, or combination}

The results of our meta-analysis suggested an improvement in FMD values after ingestion of cocoa drink, chocolate, or a combination of both $(1.17 \%$, 95\% CI: $0.76 \%, 1.57 \%, 18$ treatments, 367 participants) (Fig. 2). The high $I^{2}$ value $\left(I^{2}=83.3 \%\right)$ indicates that most of the variability across studies is due to heterogeneity rather than chance. Studies with cocoa drink intervention presented an average FMD improvement of $1.13 \%$ (CI of $0.78 \%$ and $1.48 \%$ ) while studies with chocolate showed an FMD improvement of $0.84 \%$ (CI of $0.19 \%$ and $1.50 \%$ ). Studies with cocoa drink intervention had a lower heterogeneity than those with chocolate intervention $\left(I^{2}=63.7 \%\right.$ vs. $\left.I^{2}=81.6 \%\right)$. The only study with a combination of cocoa drink plus chocolate $^{22}$ showed a higher FMD improvement $(4.34 \%, 95 \%$ CI: $3.19 \%, 5.50 \%)$.

\section{Subgroup analyses and meta-regression}

Subgroup analyses categorized by interventional treatment, trial design, trial intervention duration, doses per day (from 1 to 3 ),

Table 1 Characteristic of the trials, participants, interventions and flavanols levels in this meta-analysis

\begin{tabular}{|c|c|c|c|c|c|c|c|c|c|}
\hline First author & Year & $n \mathrm{C} / n \mathrm{~T}$ & $\begin{array}{l}\text { Age } \\
\text { (years) }\end{array}$ & \% Men & Healthy status & Control & Treatment & $\begin{array}{l}\text { Duration } \\
\text { (days) }\end{array}$ & $\begin{array}{l}\Delta \text { Total } \\
\text { flavanols } \\
(\mathrm{mg})\end{array}$ \\
\hline Balzer $^{19}$ & 2008 & $20 / 21$ & $64 \pm 8.3$ & 29 & Medicated diabetic & Cocoa drink & Cocoa drink & 8,30 & 888 \\
\hline Davison $(\mathrm{A})^{20}$ & 2008 & $13 / 13$ & $45 \pm 3.5$ & 38 & Unhealthy & $\begin{array}{l}\text { Cocoa drink } \\
\text { and exercise }\end{array}$ & $\begin{array}{l}\text { Cocoa drink } \\
\text { and exercise }\end{array}$ & 84 & 866 \\
\hline Davison $(\mathrm{B})^{20}$ & 2008 & $11 / 12$ & $45 \pm 4.4$ & 30 & Unhealthy & Cocoa drink & Cocoa drink & 84 & 866 \\
\hline Esser $^{21}$ & 2014 & $41 / 41$ & $63 \pm 5$ & 100 & Overweight & Chocolate & Dark chocolate & 28 & 819 \\
\hline Farouque $^{22}$ & 2006 & $20 / 20$ & $61 \pm 8$ & 75 & CAD & $\begin{array}{l}\text { Cocoa drink }+ \\
\text { Chocolate }\end{array}$ & $\begin{array}{l}\text { Cocoa drink }+ \\
\text { Chocolate }\end{array}$ & 21,42 & 424.4 \\
\hline Flammer $^{23}$ & 2012 & $10 / 10$ & $60 \pm 10.1$ & 85 & $\mathrm{CHF}$ & Chocolate & Chocolate & 14,28 & 1248 \\
\hline Grassi $(A)^{24}$ & 2005 & $15 / 15$ & $44 \pm 7.8$ & 50 & Hypertensive & White chocolate & Chocolate & 15 & 550 \\
\hline Grassi $(B)^{24}$ & 2005 & $15 / 15$ & $34 \pm 7.6$ & 47 & Normotensive & White chocolate & Chocolate & 15 & 550 \\
\hline Grassi $^{25}$ & 2008 & $19 / 19$ & $45 \pm 8$ & 58 & Hypertensive IGT & White chocolate & Chocolate & 15 & 1008 \\
\hline Grassi $^{26}$ & 2015 & $20 / 20$ & $54 \pm 8.9$ & 55 & Healthy & Cocoa drink & Cocoa drink & 7 & $\begin{array}{l}80,200 \\
500,800\end{array}$ \\
\hline Heiss $^{27}$ & 2010 & $16 / 16$ & $64 \pm 12$ & 81 & CAD & Cocoa drink & Cocoa drink & 30 & 750 \\
\hline Heiss $(\mathrm{A})^{28}$ & 2015 & $11 / 11$ & $26 \pm 3.2$ & 100 & Healthy & Cocoa drink & Cocoa drink & 14 & 900 \\
\hline Heiss (B) & 2015 & $10 / 10$ & $60 \pm 6$ & 100 & Healthy & Cocoa drink & Cocoa drink & 14 & 900 \\
\hline Mogollon $^{29}$ & 2013 & $20 / 22$ & $29 \pm 3.2$ & 0 & Pregnant healthy & Chocolate & Chocolate & 42,84 & 340 \\
\hline Njike $^{30}$ & 2011 & $39 / 39$ & $52 \pm 10.7$ & 15 & Overweight & Cocoa drink & $\begin{array}{l}\text { Cocoa drink } \\
\text { (with/without sugar) }\end{array}$ & 42 & 796 \\
\hline Sansone $^{31}$ & 2015 & $50 / 50$ & $45 \pm 8$ & 50 & Healthy & Cocoa drink & Cocoa drink & 28 & 900 \\
\hline Wang-Polagruto ${ }^{32}$ & 2006 & $8 / 9$ & $58 \pm 2.2$ & 0 & Hyper-cholesterolemia & Cocoa drink & Cocoa drink & 42 & 403 \\
\hline Rassaf $^{33}$ & 2016 & $25 / 24$ & $65 \pm 14$ & 73 & Haemodialysis & Cocoa drink & Cocoa drink & 30 & 900 \\
\hline
\end{tabular}

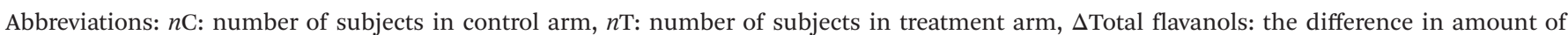

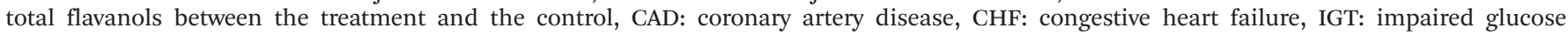
tolerance. 


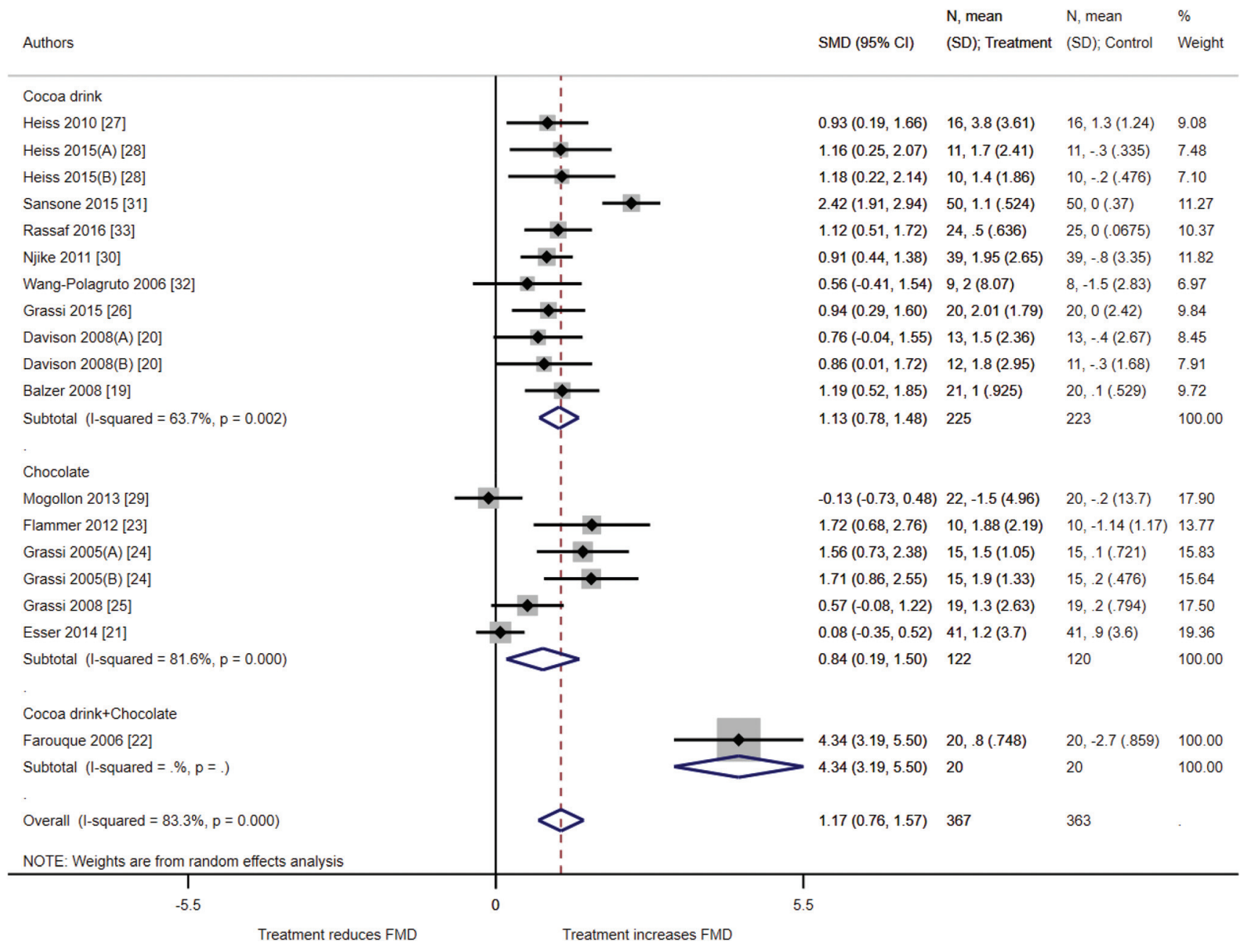

Fig. 2 Effect of cocoa drink and chocolate on flow-mediated dilation (FMD) in the included studies.

BMI, age of population treated, potential risk of bias, and source of study funding included in the meta-analysis are depicted in Table 2. The study by Farouque et al. ${ }^{22}$ combining cocoa drink plus chocolate intervention significantly differed from those studies with cocoa drink or chocolate only ( $p<$ 0.001). Studies with low risk of bias demonstrated a smaller but statistically significant effect on FMD (0.78\%, CI: $0.38 \%-$ $1.19 \%)$ than studies with moderate risk of bias $(p=0.07)$. No significant differences were observed between other subgroup categories. Remaining heterogeneity after subgroup meta-analyses showed that only trial intervention categorization accounted for a substantial amount of study heterogeneity ( $I^{2}$ was reduced from $83.3 \%$ in the overall studies to $72.6 \%$ ). Specifically, between-study heterogeneity was reduced to $0 \%$ for the following subgroups: studies with treatment duration of 7-15 days (6 studies); studies with mean subject BMI $>30 \mathrm{~kg} \mathrm{~m} \mathrm{~m}^{-2}$ (2 studies); and, studies with mean subject age ranging from 45-60 years ( 7 studies). The remaining subgroups studied did not demonstrate a major improvement in heterogeneity. No conclusions could be inferred from source of funding since only a small number of studies are grouped in each subgroup.
Since cocoa-derived products do not contain similar amounts and profiles in flavanols, subgroup analyses were performed with different dose categories for total flavanols, (-)-epicatechin and (+)-catechin respectively (Table 3). According to the categories described, maximum effect on FMD was observed in studies evaluating over $900 \mathrm{mg}$ of total flavanols per day, 50-<150 mg (-)-epicatechin per day or 20-40 mg (+)-catechin per day. Treatments with 50-<150 mg (-)-epicatechin per day showed a statistically significant difference compared with treatments containing $<50 \mathrm{mg}$ day $^{-1}$ $(p=0.05)$.

\section{Modelling dose of flavanols, (-)-epicatechin and (+)-catechin in the clinical trial treatments}

The Akaike Information Criterion (AIC) was used to define the optimal model for the relationship between the dose of total flavanols, (-)-epicatechin, (+)-catechin, and total flavanols without (-)-epicatechin and the biological effect. Fig. 3 shows the optimal models, with the degree of polynomial of 4 for total flavanols and (+)-catechin, 2 for (-)-epicatechin and 0 for total flavanols without (-)-epicatechin. An inverted $U$ shape 
Table 2 Results of subgroup analyses according to trial and participant characteristics

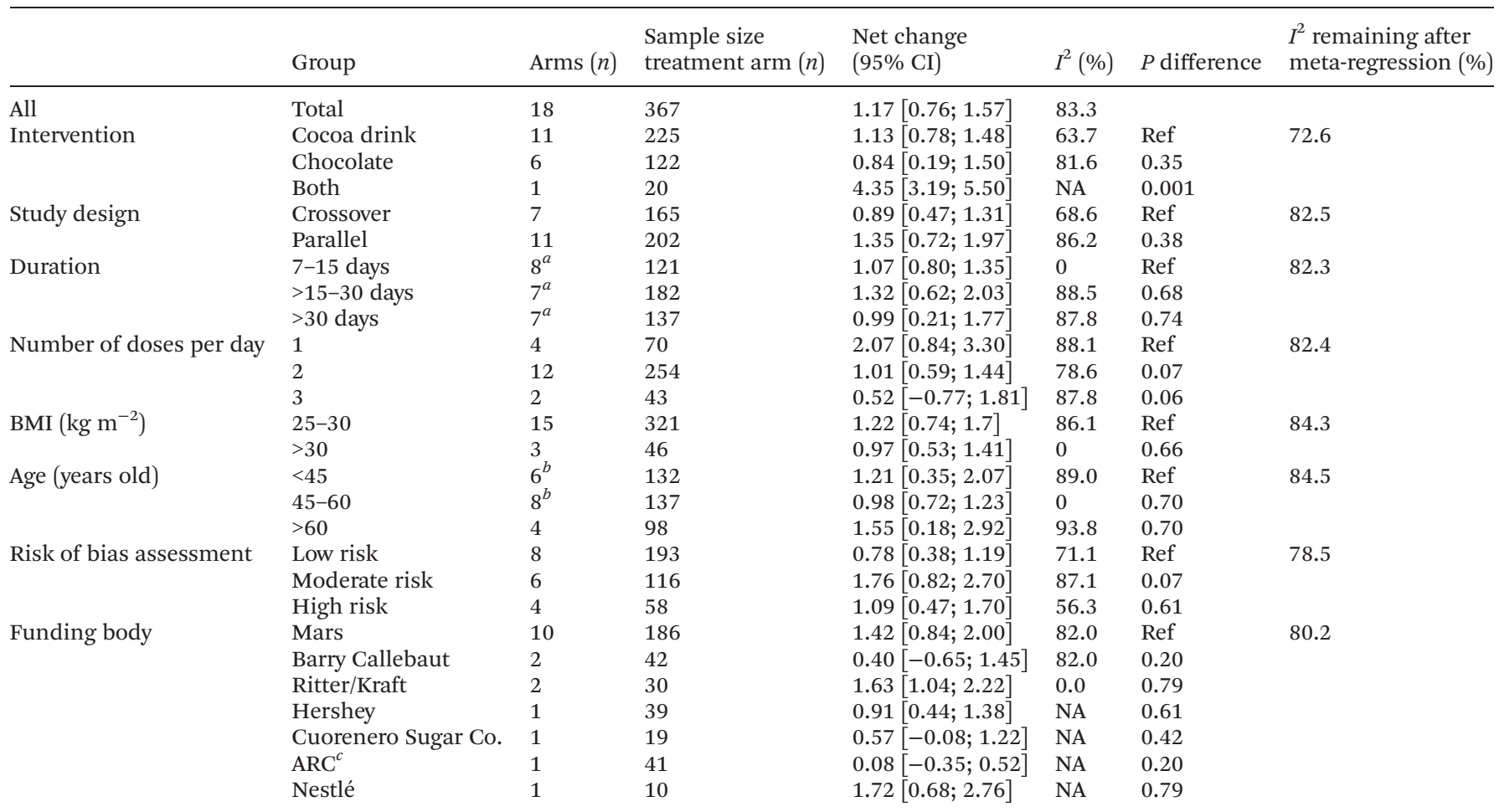

${ }^{a}$ Balzer 2008A, ${ }^{19}$ Flammer $2012 \mathrm{~B}^{23}$ ( $<15$ days), Balzer 2008B, ${ }^{19}$ Flammer 2012A, ${ }^{23}$ Farouque $2006^{22}$ ( $<15-30$ days), and Farouque $2006 \mathrm{~A}^{22}$ ( $>30$ days) are counted in different duration categories. ${ }^{b}$ Heiss $2015 \mathrm{~B}^{28}\left(45-60\right.$ years) and Heiss $2015 \mathrm{~A}^{28}(<45$ years) are counted in different age categories. ${ }^{c}$ ARC (American Research Cocoa).

Table 3 Results of subgroup analysis according to dose of total flavanols, (-)-epicatechin and (+)-catechin

\begin{tabular}{|c|c|c|c|c|c|c|c|}
\hline & Group & $\operatorname{Arms}(n)$ & $\begin{array}{l}\text { Sample size } \\
\text { treatment arm }(n)\end{array}$ & Net change (95\% CI) & $I^{2}(\%)$ & $P$ difference & $\begin{array}{l}I^{2} \text { remaining after } \\
\text { meta-regression }(\%)\end{array}$ \\
\hline \multirow{3}{*}{$\begin{array}{l}\text { All } \\
\text { Total flavanols }\end{array}$} & Total & 18 & 367 & $1.17[0.76 ; 1.57]$ & 83.3 & & \\
\hline & $800-<900 \mathrm{mg}^{\circ} \mathrm{day}^{-1}$ & $5^{a}$ & 107 & $0.72[0.25 ; 1.19]$ & 60.2 & 0.59 & \multirow{2}{*}{79.4} \\
\hline & $>900 \mathrm{mg} \mathrm{day}{ }^{-1}$ & 6 & 124 & $1.37[0.73 ; 2.01]$ & 78.1 & 0.59 & \\
\hline (-)-Epicatechin & $0-<50 \mathrm{mg}$ day $^{-1}$ & $4^{b}$ & 101 & $0.54[0.08 ; 1.00]$ & 59.5 & Ref & 75.1 \\
\hline \multirow[t]{3}{*}{$(+)$-Catechin } & $0-<20 \mathrm{mg}$ day $^{-1}$ & $8^{c}$ & 173 & $1.00[0.40 ; 1.60]$ & 84.4 & Ref & \multirow[t]{3}{*}{80.8} \\
\hline & $20-<40 \mathrm{mg} \mathrm{\textrm {day } ^ { - 1 }}$ & $8^{c}$ & 147 & $1.46[0.82 ; 2.10]$ & 82.0 & 0.33 & \\
\hline & $>40 \mathrm{mg} \mathrm{day}^{-1}$ & $5^{c}$ & 107 & $0.72[0.25 ; 1.19]$ & 60.2 & 0.61 & \\
\hline
\end{tabular}

${ }^{a}$ Grassi 2015D (800-<900 $\left.\mathrm{mg}^{-1} \mathrm{y}^{-1}\right)$, Grassi 2015A, Grassi 2015B and Grassi 2015C (500-<800 mg day $\left.{ }^{-1}\right)$ are counted in different dose categories. ${ }^{b}$ Grassi 2015D ( $\left.>40 \mathrm{mg} \mathrm{day}^{-1}\right)$, Grassi 2015C $\left(20-40 \mathrm{mg}\right.$ day $\left.{ }^{-1}\right)$, Grassi 2015A and Grassi 2015B $\left(0-<20 \mathrm{mg}^{-1 a{ }^{-1}}\right)$ are counted in different dose categories. ${ }^{c}$ Grassi $2015 \mathrm{D}\left(>150 \mathrm{mg}\right.$ day $\left.{ }^{-1}\right)$, Grassi 2015C $\left(50-150 \mathrm{mg}\right.$ day $\left.{ }^{-1}\right)$, Grassi 2015A and Grassi $2015 \mathrm{~B}\left(0-<50 \mathrm{mg}\right.$ day $\left.{ }^{-1}\right)$ are counted in different dose categories.

relationship can be seen between total flavanols, (-)-epicatechin, and (+)-catechin with FMD, with a maximum vascular effect for $710 \mathrm{mg}$ total flavanols (FMD 2.5\%), $95 \mathrm{mg}(-)$-epicatechin (FMD 3\%) and $25 \mathrm{mg}$ (+)-catechin (FMD 2.5\%). A physiologically relevant effect size of $1 \%$ increase in FMD could be achieved with doses ranging from 150 to $1000 \mathrm{mg}$ of total flavanols, 40 to $140 \mathrm{mg}$ of (-)-epicatechin, or 15 to $38 \mathrm{mg}$ of $(+)$-catechin. The curve for total flavanols without (-)-epicate- chin depicted a different shape, suggesting that (-)-epicatechin content could be responsible for an important part of the relationship with FMD. Altogether, the results indicate that the (-)-epicatechin dose in the different interventional products plausibly drives the biological effect over the vasculature. Other ingredients also present in the treatments, such as theobromine or carbohydrates, could not be plotted since insufficient data points were available or there was no specific relationship. 

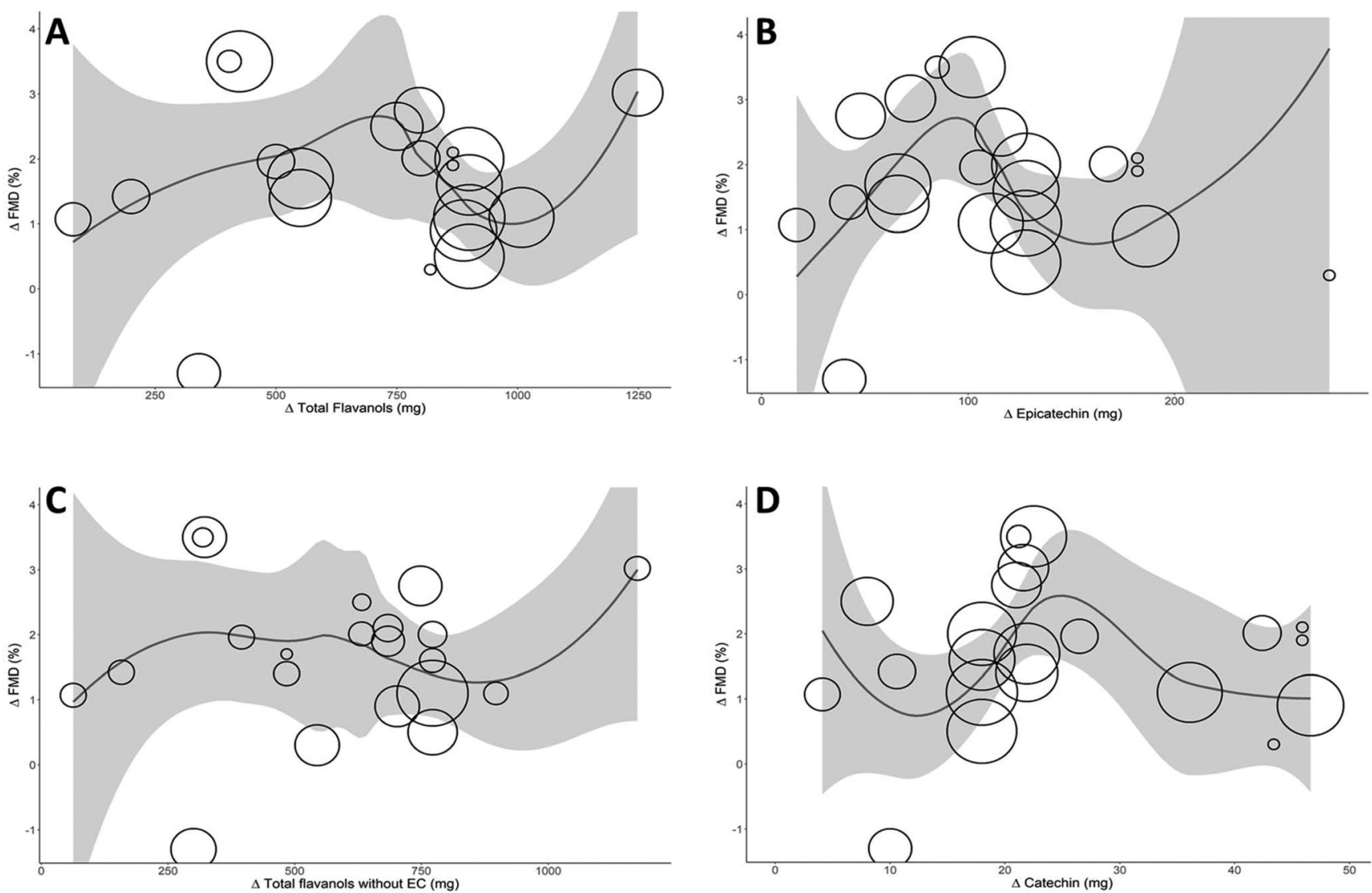

Fig. 3 Modelling of difference between intervention treatment FMD minus control treatment FMD [delta FMD ( $\triangle F M D)$ ] vs. (a) difference between total flavanol in intervention treatment minus total flavanol control treatment ( $\Delta$ Total flavanols); (b) difference between (-)-epicatechin in intervention treatment minus (-)-epicatechin control treatment ( $\Delta$ Epicatechin); (c) difference between total flavanol without ( - )-epicatechin in intervention treatment minus total flavanol without $(-)$-epicatechin control treatment ( $\Delta$ Total flavanols without $\mathrm{EC})$; (d) difference between $(+)$-catechin in intervention treatment minus $(+)$-catechin control treatment ( $\Delta$ Catechin).

\section{Sensitivity analyses and publication bias}

Sensitivity analyses with fixed-effects models did not differ significantly from those observed in random-effects models (data not shown). Removing studies individually did not substantially change the differences in effect on FMD, with effect estimates ranging from $0.96 \%$ ( $95 \%$ CI: $0.69,1.24 \%$ ) by removing Farouque $2006 \mathrm{~A}^{22}$ to $1.14 \%$ (95\% CI: $0.82,1.46 \%$ ) by removing Mogollon 2013B ${ }^{29}$ (see ESI Fig. $1 \dagger$ ). Egger's test $(p=0.08$ ), Begg's test $(p=0.03)$, and the funnel plot indicated that the meta-analysis may be affected by publication bias (see ESI Fig. $2 \dagger$ ). Overall risk of bias for each of the publications can be seen in ESI Table 1. $\dagger$

\section{Discussion}

The aim of this investigation was to assess the current scientific evidence on the relationship between cocoa flavanols and endothelial function, measured by FMD, and to evaluate the potential main bioactive and optimal dose from cocoa/chocolate to affect endothelial function. Our meta-analysis included 15 clinical trials, 18 intervention arms with 730 participants using interventions of at least 7 days with cocoa drink, chocolate, or both. The results showed an FMD improvement of $1.08 \%$ as a measurement of vascular endothelial function. These findings support a previous meta-analysis that described an overall effect of 1.34\% FMD (CI: 1.00\%, 1.68\%) for studies longer than 2 weeks ( 9 clinical trials with 545 participants). ${ }^{3}$

Brachial FMD has been inversely associated with future cardiovascular events. ${ }^{34-36}$ Meta-analyses have suggested a $13 \%$ risk reduction of cardiovascular events with a $1 \%$ increase of FMD. ${ }^{37,38}$ Hence, the improvement of FMD values observed in our meta-analysis supports the notion that chronic consumption of cocoa-derived products could decrease the overall risk of cardiovascular diseases (CVD). Importantly, there is a stronger association between FMD and CVD risk for diseased populations than for asymptomatic populations. ${ }^{38}$ Therefore, even small increases in FMD could be relevant for diseased populations.

A further subgroup analysis with different levels of total flavanols, (-)-epicatechin or (+)-catechin in the intervention treatments showed significantly higher FMD improvement with treatments containing between 50 to $150 \mathrm{mg}$ of (-)-epicatechin. Interestingly, interventions with lower or higher doses of 
(-)-epicatechin showed a lesser effect on improving endothelial function. Modelling the dose of flavanols (-)-epicatechin and (+)-catechin in the trial treatments depicted an inverted $U$ shape with maximum effect observed at $710 \mathrm{mg}$ total flavanols, $95 \mathrm{mg}$ (-)-epicatechin and $25 \mathrm{mg}$ $(+)$-catechin. It is worth noting that (-)-epicatechin and $(+)$-catechin levels are correlated in the test treatments used in the analysed studies $(r=0.78)$. Therefore, we cannot infer the individual impact of each of them on improving endothelial function.

Acute clinical trials demonstrating improvement in FMD outcomes with other high-polyphenol-containing foods described an inverted $\mathrm{U}$ shape relationship similar to that observed with cocoa polyphenols in this analysis. For instance, single consumption of several doses of blueberry drinks (from freeze-dried powder) showed a dose response with a maximum effect at $766 \mathrm{mg}$ of polyphenols. ${ }^{39}$ Higher doses containing 1278 or $1791 \mathrm{mg}$ of polyphenols did not show a higher impact on FMD values. Another study with different doses of cranberry juice demonstrated an improvement on FMD. ${ }^{40}$ An inverted $U$ shape was observed with the dose containing $1238 \mathrm{mg}$ of total polyphenols being the most effective.

The idea that there is an optimal dose range of bioactives that would result in an optimal and physiologically relevant health effect could have large consequences for future dietary recommendations. In addition to the notion that higher concentrations do not always bring more benefits, the seasonal variation and the influence of processing on the polyphenol content in flavanol-rich foods could have significant impact on the health benefits that may be expected from the consumption of these foods. It is all the most important since, contrary to micronutrients (e.g. vitamins and minerals), these bioactives are not stored within the human body.

Our meta-analysis has some inherent limitations. Firstly, although 15 studies (with 18 study arms) were included, there is high variability in the health status of the participants as well as in the duration of the treatments (from 7 to 84 days). Heterogeneity in treatment duration and other study characteristics was investigated through subgroup analyses. However, due to varying definitions of health status used in individual studies (e.g. diabetic, hypertensive, overweight, etc.), it is impossible to conduct subgroup analyses by health status except via BMI categories. Furthermore, a number of these studies did not describe the specific flavanol levels (i.e (-)-epicatechin or (+)-catechin) in the treatments. Consequently, the levels of flavanols in these cases had to be estimated from other publications. High heterogeneity was also found in the data analysed $\left(I^{2}=80 \%\right)$. Subgroup analysis did not show a significant decrease in heterogeneity except for a few subgroups. Therefore, the high variability in trial characteristics, the need to estimate the levels of specific flavanols in treated groups, and the heterogeneity of primary data used, could reduce the confidence of recommendations about the effect of consuming cocoa-derived products to improve the vascular endothelial function.
In summary, our meta-analysis of randomized clinical trials confirmed previous findings that consumption of flavanol-rich foods such as cocoa drinks or chocolate could significantly improve endothelial function. In addition, the modelling of total flavanols, (-)-epicatechin and (+)-catechin content in the treatments as a function of the improvement on FMD suggests a limited range of optimal dose. However, further clinical trials, particularly with a wide range of doses, are warranted to confirm the effective dose of flavanols with optimal cardiovascular-related benefits.

\section{Funding}

This work was supported financially by Nestec Ltd, a subsidiary of Nestlé Ltd.

\section{Author contributions}

LAG, YS and DZ co-designed the scope of the review and performed the literature search. YS and AdC performed the metaanalysis and subgroup analyses of the data. LAG and YS drafted the article. All authors reviewed, edited and approved the final version of the manuscript for submission.

\section{Data availability}

All data generated during this study are included in the published article (and its ESI $\dagger$ ).

\section{Abbreviations}
FMD Flow-mediated dilation
RCTs Randomized clinical trials
SD Standard deviations
SEs Standard errors
CIs Confidence intervals
AIC Akaike information criterion
CVD Cardiovascular diseases

\section{Conflicts of interest}

YS, AdC and LAG are employees of the Nestlé Research Centre, Singapore. DZ is an employee of Nestlé Research in Switzerland.

\section{Acknowledgements}

Editorial support for the manuscript was obtained from Rosalind McKinnon at RAPID Consulting GmbH (Laufen, Switzerland) and funded by Nestlé. 


\section{References}

1 X. Lin, I. Zhang, A. Li, J. E. Manson, H. D. Sesso, L. Wang and S. Liu, Cocoa Flavanol Intake and Biomarkers for Cardiometabolic Health: A Systematic Review and MetaAnalysis of Randomized Controlled Trials, J. Nutr., 2016, 146, 2325-2333.

2 A. Buitrago-Lopez, J. Sanderson, L. Johnson, S. Warnakula, A. Wood, E. Di Angelantonio and O. H. Franco, Chocolate consumption and cardiometabolic disorders: systematic review and meta-analysis, Br. Med. J., 2011, 343, d4488.

3 L. Hooper, C. Kay, A. Abdelhamid, P. A. Kroon, J. S. Cohn, E. B. Rimm and A. Cassidy, Effects of chocolate, cocoa, and flavan-3-ols on cardiovascular health: a systematic review and meta-analysis of randomized trials, Am. J. Clin. Nutr., 2012, 95, 740-751.

4 L. Hooper, P. A. Kroon, E. B. Rimm, J. S. Cohn, I. Harvey, K. A. Le Cornu, J. J. Ryder, W. L. Hall and A. Cassidy, Flavonoids, flavonoid-rich foods, and cardiovascular risk: a meta-analysis of randomized controlled trials, Am. J. Clin. Nutr., 2008, 88, 38-50.

5 D. Mozaffarian, Dietary and Policy Priorities for Cardiovascular Disease, Diabetes, and Obesity: A Comprehensive Review, Circulation, 2016, 133, 187-225.

6 J. I. Ottaviani, C. Heiss, J. P. E. Spencer, M. Kelm and H. Schroeter, Recommending flavanols and procyanidins for cardiovascular health: Revisited, Mol. Aspects Med., 2018, 61, 63-75.

7 J. I. Ottaviani, C. Kwik-Uribe, C. L. Keen and H. Schroeter, Intake of dietary procyanidins does not contribute to the pool of circulating flavanols in humans, Am. J. Clin. Nutr., 2012, 95, 851-858.

8 J. I. Ottaviani, G. Borges, T. Y. Momma, J. P. Spencer, C. L. Keen, A. Crozier and H. Schroeter, The metabolome of [2-(14)C](-)-epicatechin in humans: implications for the assessment of efficacy, safety, and mechanisms of action of polyphenolic bioactives, Sci. Rep., 2016, 6, 29034.

9 C. E. Mills, A. Flury, C. Marmet, L. Poquet, S. F. Rimoldi, C. Sartori, E. Rexhaj, R. Brenner, Y. Allemann, D. Zimmermann, G. R. Gibson, D. S. Mottram, M. J. OrunaConcha, L. Actis-Goretta and J. P. Spencer, Mediation of coffee-induced improvements in human vascular function by chlorogenic acids and its metabolites: Two randomized, controlled, crossover intervention trials, Clin. Nutr., 2017, 36(6), 1520-1529.

10 H. Schroeter, C. Heiss, J. Balzer, P. Kleinbongard, C. L. Keen, N. K. Hollenberg, H. Sies, C. Kwik-Uribe, H. H. Schmitz and M. Kelm, (-)-Epicatechin mediates beneficial effects of flavanol-rich cocoa on vascular function in humans, Proc. Natl. Acad. Sci. U. S. A., 2006, 103, 1024-1029.

11 J. I. Dower, J. M. Geleijnse, L. Gijsbers, P. L. Zock, D. Kromhout and P. C. Hollman, Effects of the pure flavonoids epicatechin and quercetin on vascular function and cardiometabolic health: a randomized, double-blind, placebo-controlled, crossover trial, Am. J. Clin. Nutr., 2015, 101, 914-921.
12 J. P. Higgins, D. G. Altman, P. C. Gotzsche, P. Juni, D. Moher, A. D. Oxman, J. Savovic, K. F. Schulz, L. Weeks, J. A. Sterne, G. Cochrane Bias Methods and G. Cochrane Statistical Methods, The Cochrane Collaboration's tool for assessing risk of bias in randomised trials, Br. Med. J., 2011, 343, d5928.

13 F. Curtin, D. Elbourne and D. G. Altman, Meta-analysis combining parallel and cross-over clinical trials. III: The issue of carry-over, Stat. Med., 2002, 21, 2161-2173.

14 J. P. Higgins and S. G. Thompson, Quantifying heterogeneity in a meta-analysis, Stat. Med., 2002, 21, 1539-1558.

15 H. Wickham, ggplot2: Elegant Graphics for Data Analysis, Springer-Verlag, New York, 1st edn, 2009.

16 J. A. Sterne and M. Egger, Funnel plots for detecting bias in meta-analysis: guidelines on choice of axis, J. Clin. Epidemiol., 2001, 54, 1046-1055.

17 C. B. Begg and M. Mazumdar, Operating characteristics of a rank correlation test for publication bias, Biometrics, 1994, 50, 1088-1101.

18 M. Egger, G. Davey Smith, M. Schneider and C. Minder, Bias in meta-analysis detected by a simple, graphical test, Br. Med. J., 1997, 315, 629-634.

19 J. Balzer, T. Rassaf, C. Heiss, P. Kleinbongard, T. Lauer, M. Merx, N. Heussen, H. B. Gross, C. L. Keen, H. Schroeter and M. Kelm, Sustained benefits in vascular function through flavanol-containing cocoa in medicated diabetic patients a double-masked, randomized, controlled trial, J. Am. Coll. Cardiol., 2008, 51, 2141-2149.

20 K. Davison, A. M. Coates, J. D. Buckley and P. R. Howe, Effect of cocoa flavanols and exercise on cardiometabolic risk factors in overweight and obese subjects, Int. J. Obes., 2008, 32, 1289-1296.

21 D. Esser, M. Mars, E. Oosterink, A. Stalmach, M. Muller and L. A. Afman, Dark chocolate consumption improves leukocyte adhesion factors and vascular function in overweight men, FASEB J., 2014, 28, 1464-1473.

22 H. M. Farouque, M. Leung, S. A. Hope, M. Baldi, C. Schechter, J. D. Cameron and I. T. Meredith, Acute and chronic effects of flavanol-rich cocoa on vascular function in subjects with coronary artery disease: a randomized double-blind placebo-controlled study, Clin. Sci., 2006, 111, 71-80.

23 A. J. Flammer, I. Sudano, M. Wolfrum, R. Thomas, F. Enseleit, D. Periat, P. Kaiser, A. Hirt, M. Hermann, M. Serafini, A. Leveques, T. F. Luscher, F. Ruschitzka, G. Noll and R. Corti, Cardiovascular effects of flavanol-rich chocolate in patients with heart failure, Eur. Heart J., 2012, 33, 2172-2180.

24 D. Grassi, S. Necozione, C. Lippi, G. Croce, L. Valeri, P. Pasqualetti, G. Desideri, J. B. Blumberg and C. Ferri, Cocoa reduces blood pressure and insulin resistance and improves endothelium-dependent vasodilation in hypertensives, Hypertension, 2005, 46, 398-405.

25 D. Grassi, G. Desideri, S. Necozione, C. Lippi, R. Casale, G. Properzi, J. B. Blumberg and C. Ferri, Blood pressure is reduced and insulin sensitivity increased in glucose-intol- 
erant, hypertensive subjects after 15 days of consuming high-polyphenol dark chocolate, J. Nutr., 2008, 138, 16711676.

26 D. Grassi, G. Desideri, S. Necozione, P. di Giosia, R. Barnabei, L. Allegaert, H. Bernaert and C. Ferri, Cocoa consumption dose-dependently improves flow-mediated dilation and arterial stiffness decreasing blood pressure in healthy individuals, J. Hypertens., 2015, 33, 294-303.

27 C. Heiss, S. Jahn, M. Taylor, W. M. Real, F. S. Angeli, M. L. Wong, N. Amabile, M. Prasad, T. Rassaf, J. I. Ottaviani, S. Mihardja, C. L. Keen, M. L. Springer, A. Boyle, W. Grossman, S. A. Glantz, H. Schroeter and Y. Yeghiazarians, Improvement of endothelial function with dietary flavanols is associated with mobilization of circulating angiogenic cells in patients with coronary artery disease, J. Am. Coll. Cardiol., 2010, 56, 218-224.

28 C. Heiss, R. Sansone, H. Karimi, M. Krabbe, D. Schuler, A. Rodriguez-Mateos, T. Kraemer, M. M. Cortese-Krott, G. G. Kuhnle, J. P. Spencer, H. Schroeter, M. W. Merx, M. Kelm and E. U. t. F. P. Flaviola Consortium, Impact of cocoa flavanol intake on age-dependent vascular stiffness in healthy men: a randomized, controlled, double-masked trial, Age, 2015, 37, 9794.

29 J. A. Mogollon, E. Bujold, S. Lemieux, M. Bourdages, C. Blanchet, L. Bazinet, C. Couillard, M. Noel and S. Dodin, Blood pressure and endothelial function in healthy, pregnant women after acute and daily consumption of flavanolrich chocolate: a pilot, randomized controlled trial, Nutr. J., 2013, 12, 41.

30 V. Y. Njike, Z. Faridi, K. Shuval, S. Dutta, C. D. Kay, S. G. West, P. M. Kris-Etherton and D. L. Katz, Effects of sugar-sweetened and sugar-free cocoa on endothelial function in overweight adults, Int. J. Cardiol., 2011, 149, 83-88.

31 R. Sansone, A. Rodriguez-Mateos, J. Heuel, D. Falk, D. Schuler, R. Wagstaff, G. G. Kuhnle, J. P. Spencer, H. Schroeter, M. W. Merx, M. Kelm, C. Heiss and E. U. t. F. P. Flaviola Consortium, Cocoa flavanol intake improves endothelial function and Framingham Risk Score in healthy men and women: a randomised, controlled, double-masked trial: the Flaviola Health Study, Br. J. Nutr., 2015, 114, 1246-1255.

32 J. F. Wang-Polagruto, A. C. Villablanca, J. A. Polagruto, L. Lee, R. R. Holt, H. R. Schrader, J. L. Ensunsa, F. M. Steinberg, H. H. Schmitz and C. L. Keen, Chronic consumption of flavanol-rich cocoa improves endothelial function and decreases vascular cell adhesion molecule in hypercholesterolemic postmenopausal women, J. Cardiovasc. Pharmacol., 2006, 47(Suppl 2), S177-S186, discussion S206-9.

33 T. Rassaf, C. Rammos, U. B. Hendgen-Cotta, C. Heiss, W. Kleophas, F. Dellanna, J. Floege, G. R. Hetzel and M. Kelm, Vasculoprotective Effects of Dietary Cocoa Flavanols in Patients on Hemodialysis: A Double-Blind, Randomized, Placebo-Controlled Trial, Clin. J. Am. Soc. Nephrol., 2016, 11, 108-118.

34 M. L. Muiesan, M. Salvetti, A. Paini, C. Monteduro, G. Galbassini, P. Poisa, E. Porteri, C. Agabiti-Rosei, V. Paderno, E. Belotti, D. Rizzoni, M. Castellano and E. Agabiti-Rosei, Prognostic role of flow-mediated dilatation of the brachial artery in hypertensive patients, J. Hypertens., 2008, 26, 1612-1618.

35 G. Brevetti, A. Silvestro, V. Schiano and M. Chiariello, Endothelial dysfunction and cardiovascular risk prediction in peripheral arterial disease: additive value of flowmediated dilation to ankle-brachial pressure index, Circulation, 2003, 108, 2093-2098.

36 S. D. Katz, K. Hryniewicz, I. Hriljac, K. Balidemaj, C. Dimayuga, A. Hudaihed and A. Yasskiy, Vascular endothelial dysfunction and mortality risk in patients with chronic heart failure, Circulation, 2005, 111, 310-314.

37 Y. Inaba, J. A. Chen and S. R. Bergmann, Prediction of future cardiovascular outcomes by flow-mediated vasodilatation of brachial artery: a meta-analysis, Int. J. Cardiovasc. Imaging, 2010, 26, 631-640.

38 R. T. Ras, M. T. Streppel, R. Draijer and P. L. Zock, Flowmediated dilation and cardiovascular risk prediction: a systematic review with meta-analysis, Int. J. Cardiol., 2013, 168, 344-351.

39 A. Rodriguez-Mateos, C. Rendeiro, T. Bergillos-Meca, S. Tabatabaee, T. W. George, C. Heiss and J. P. Spencer, Intake and time dependence of blueberry flavonoidinduced improvements in vascular function: a randomized, controlled, double-blind, crossover intervention study with mechanistic insights into biological activity, Am. J. Clin. Nutr., 2013, 98, 1179-1191.

40 A. Rodriguez-Mateos, R. P. Feliciano, A. Boeres, T. Weber, C. N. Dos Santos, M. R. Ventura and C. Heiss, Cranberry (poly)phenol metabolites correlate with improvements in vascular function: A double-blind, randomized, controlled, dose-response, crossover study, Mol. Nutr. Food Res., 2016, 60, 2130-2140. 